\title{
Identity Recognition Algorithm Based on Static Gait Geometrical and Pressure Data
}

\author{
Kun Wang ${ }^{1}$, Yiwu Qian ${ }^{1}$, Jinmo $\mathrm{Wu}^{1, *}$, and Xiaoyong Liu ${ }^{1}$ \\ ${ }^{1}$ Hefei Institute for Public Safety Research, Tsinghua University, Hefei, Anhui, 230601, P.R.CHINA
}

\begin{abstract}
Currently, pattern recognition technique is widely applied in human identity recognition while there are shortages remaining in most kinds of such techniques. In order to overcome these problems, a novel algorithm is proposed to apply in identity recognition course in new field-static gait recognition field. Two combined features are going for static gait recognition: the distance between each part of center pressure and overall foot and the side length of outline triangle of human foot. Then through the comparison of different classifier, the best course for recognition can be obtained.
\end{abstract}

\section{Introduction}

With the development of science and technology, people tend to pay more attention on the convenience and effectiveness in daily life ${ }^{[1]}$. No matter in security check places such as airport, customs, important conference, or in Entry-Exit procedure of some financial places like exchequer and safe-deposit vault, identification and identity authentication has become a vital prerequisite. In these situations, more and more rapid and effective recognition courses and systems come into beings. Some identification of physics fields such as identity card, key and several of dissimilar concrete identification substances have already been used for many decades ${ }^{[2]}$. However, these identifications all have certain shortcomings: identification substances maybe get lost or even be stolen on illegal purposes so that they cannot meet the requirement of the advanced security system. In recent years, growing numbers of identity recognitions based on the biological information are used in recognition fields ${ }^{[3]}$. Some of them have already been applied in safety system of company and house guard such as fingerprint identification, iris recognition, face recognition and so forth. Besides, some other recognition still stays in the stages of developing and modifying like the gene recognition, DNA recognition ${ }^{[4]}$. Compared with traditional identification recognition, these feature recognition technologies based on biological information have the advantages of convenient carrying-over, counterfeit difficulty and without-memory. For the moment, nonetheless, almost every recognition system has to overcome many limitations ${ }^{[5]}$. For fingerprint identification, perhaps it will be challenged by the clarity of finger texture features. For iris recognition, it needs expensive costs although has great precision. Face recognition will be limited by the emotions of human's faces. In addition, gene recognition and DNA recognition remind to understand the examination of times and society. Furthermore, different recognition system can only be used in dissimilar place ${ }^{[6]}$. In addition, the acquisition of these biology information features also requires examinees to be cooperative. Modern medical research shows the nuance of bonemuscle system of human body has its uniqueness, which seems to be promising. Under these circumstances, static gait recognition is proposed to apply in certain place, like indoor sport court, gymnasium and entertainment places ${ }^{[7]}$. Therefore, through the analysis of static pressure data of human feet, this method can not only avoid being influenced by external factors, but also has a high recognition efficiency of different feet print. Besides, no matter for the physical feature recognition, the current biological feature recognition or even the contacting identity recognition, they all belong to short range recognition ${ }^{[8]}$. And these recognition methods do not suit some certain places which need secretive and long-range recognition course. Based on these situations, a new identity recognition which takes advantage of gait touching feature is proposed.

Gait recognition is a kind of new recognition technology which contains the technologies, like computer vision, pattern recognition and image sequence in computer and signal processing and has great application prospect ${ }^{[9]}$. Thus, many scholars of foreign or domestic pay much attention on this course. Foreign countries already have advanced development before, especially after September 11th in the USA, the application of gait recognition in video surveillance system field had attracted more attention in every nation. Recently, the identity recognitions based on gait feature have already become a key research project for scientific researchers and renowned research institutions. For example, Begg attempted to bring the Support Vector Machine into gait recognition filed so as to identify samples automatically and achieved success. In addition, MIT (Massachusetts Institute of Technology) and Carnegie Mellon University in the USA have also made a great progress in gait recognition field based on

\footnotetext{
* Corresponding author: wujinmo@tsinghua-hf.edu.cn
} 
computer vision ${ }^{[10]}$. Jung used the track of pressure center to describe the dynamic change of foot pressure and directivity index to indicate the direction change of the track of pressure center to extract classification feature. Compared with foreign nations, China has a later and slower development. Qian Gang extracted the track of pressure center from foot pressure distribution to deal with identity recognition. Lei Hang and Ping Jianxi extracted 23 geometric feature parameters, 12 regional shape characteristic parameters and 6 contour shape characteristic parameters to identify 266 people and obtained a better result ${ }^{[11]}$.

Contrasted to some other recognition courses in physical and biological fields, the advantage of this study is: Different people have dissimilar pressure distribution when touching ground during their walking [12]. Medical research shows that the nuisance of body's skeletal muscle system and the difference of walking style and body type provide the uniqueness of distribution of people's footprints. So, in order to make the purpose of recognition, gait touching feature can be obtained by dealing with body's pressure distribution. Furthermore, combing with the recognition method using Support Vector Machine (SVM) classifier, this course can accomplish the identity recognition of 50 testers ${ }^{[13]}$. To some extent, the course overcomes the shortages in the recognitions of physical and biological fields, as well as avoids the difficulty in feature extraction of some certain recognition methods. For the reason that the research of static gait recognition methods are staying at start-up level, this paper simply makes an attempt to deal with the static gait data of testers. The potential application is an auxiliary and supplement of current recognition technology and can also go into a deep level so as to become a sheltered identity recognition technology ${ }^{[14]}$.

\section{Feature extraction and recogtion of gait data}

The testing square of pressure distribution beta consists of 1600 sensors which arrange in the pattern of array change design is $40 \mathrm{~cm} \times 40 \mathrm{~cm}$, forming 40 rows $\times 40$ columns using 100 leads. Figure 1 shows pressureplotting of the footprints obtaining in pressure distribution beta, different colors represent different value of pressure and the unit of the value is obtained by the quotient of weight of testers and the number of grid squares of testers' foot pressure. The gray area in the picture represents the area of touching place without pressure value and the white area represents the area of untouched place. The values pressure of gray and white areas are both 0 , in addition, the histogram of the distribution of pressure is showed in Figure 1.

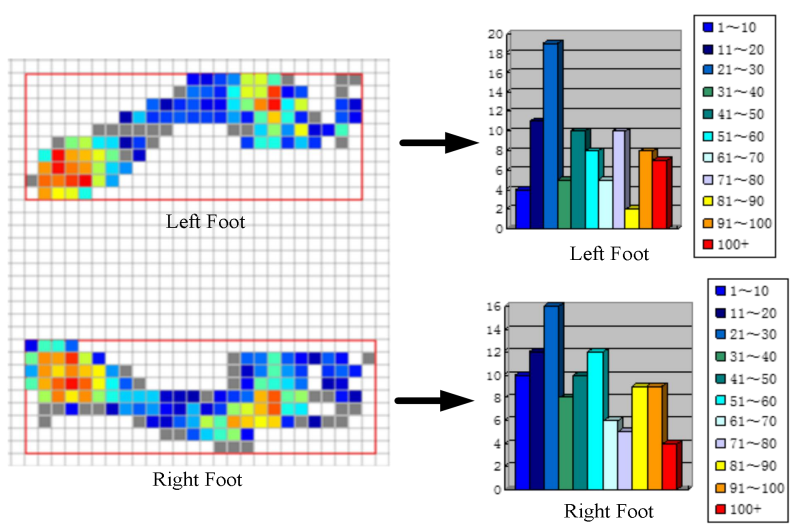

Fig. 1. Pressure distribution and histogram

With the pressure distribution beta, the data of testers' foot pressure distribution which are depicted by static pressure matrix can be acquired. The element values in the matrix represent the values of present foottouching points which are showed in Figure 2. In addition, there are many zero value points in the static pressure matrix, which are presented by gray and white squares and also all-zero lines and all-zero columns of the white parts of the matrix can be eliminated purposely. After that, the real footprints of testers will be obtained.

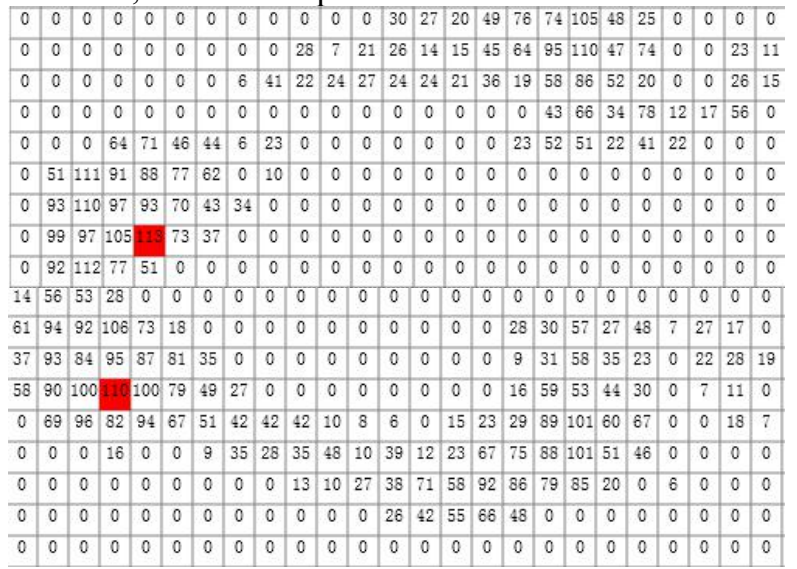
Fig. 1. Static gait pressure matrix

\section{Data processing and classifier}

\subsection{Preprocessing of static Gait pressure matrix}

During the course of collecting gait data, the right directions of testers' foot are hard to be tested for testers' dissimilar standing posture. In order to change the directions of different gait pressure so as to make all testers' gait pressure matrix towards the same direction to obtain the most accurate testing result, the preprocessing of gait pressure matrix is necessary to the recognition course. The preprocessing course mainly talks about the issue of rotation-invariant processing whose concrete course is described as follows:

According to the structural feature of human feet, the overall pressure point of human foot is located at the geometric center of the sole, that is to say, the heel, roughly. Then the maximum distance between the overall pressure point and other points in the gait pressure matrix can be obtained. In most cases, the other 
point which can get the maximum is located at the area of big toe part of the matrix. Thus, there is an angle between the line connecting the two points and the horizontal line. Then rotate the obtained line towards the horizontal line about this certain angle so as to make sure the directions of testers' feet toward the same direction.

\subsection{Gait touching feature}

In this recognition course, in order to increase the accuracy of experiment, two features are combined to perform the course ${ }^{[15]}$. The first one is based on the distances of overall-pressure point to two partial pressure point. Definitely, there is an overall-pressure point $P_{c}\left(X_{c}, Y_{c}\right)$ existing on human's foot. And also the whole sole can be divided into two similar parts of partial area if they are segmented equally, that is, the back half and the front half of sole. Certainly, the front half of sole includes toe area. For the sake of the two parts, the static gait pressure matrix will be divided in the $50 \%$ of the position of the whole matrix. Hence, two parts of partial area can be obtained. In addition, each one has its pressure points. In this case, one overall-pressure point $P_{c}\left(X_{c}, Y_{c}\right)$ in Eq.1 and Eq.2, and two partial points $P_{1}\left(X_{1}, Y_{1}\right)$ (the pressure point of front sole) and $P_{2}\left(X_{2}, Y_{2}\right)$ (the pressure of back sole) will be found to acquire the two distances from the overall-pressure to the two partial pressure points.

$$
\begin{gathered}
X_{c}=\sum_{i=1}^{n}\left(X_{i} \times P_{i}\right) / \sum_{i=1}^{n} P_{i} \\
Y_{c}=\sum_{i=1}^{n}\left(Y_{i} \times P_{i}\right) / \sum_{i=1}^{n} P_{i}
\end{gathered}
$$

The two distances can be marked as $d_{1}$ and $d_{2}$ from Eq. 3 and Eq.4.

$$
\begin{aligned}
& d_{1}=\sqrt{\left(Y_{c}-Y_{1}\right)^{2}+\left(X_{c}-X_{1}\right)^{2}} \\
& d_{2}=\sqrt{\left(Y_{c}-Y_{2}\right)^{2}+\left(X_{c}-X_{2}\right)^{2}}
\end{aligned}
$$

The second feature can be called distances of three outline points. Human foot has its outline which is consisted of countless points among which three points can be selected ${ }^{[16]}$. These three points are chosen with reason, not each point can represent the outline feature of human foot. Hence, three different points are selected to constitute the second feature of human foot. Although human foot may has similar form, the outlines of footprints of different testers have the dissimilar appearance due to the difference of testers' weight, habit station appearance, foot arch and so forth. Due to these different factors, some special points can be selected to represent the characteristic of sole information in the static gait pressure matrix. For the inner side of sole, two points $P_{3}$ and $P_{4}$ can be extracted. $P_{3}$ is extracted from the front part of inner side of sole and located at the most inner points of static gait pressure matrix. Exactly, for almost every tester, $P_{3}$ exists in the position of protruding bone near big toe due to the physiology structure of human's foot. If there is only one point lying in the most inner position, this point is taken as $P_{3}$ and if there are more than one point lies in that position, the midpoint of these pints is taken as $P_{3}$, shown in Eq.5. The y-coordinate of $P_{3}$ can be obtained from the average of the $y$-coordinate of the points lying in the most inner row of static gait pressure matrix and $\mathrm{x}$-coordinate is the row of random $\mathrm{x}$-coordinate of these points because they lie in the same row after the preprocessing of static gait pressure matrix, shown in Eq.6.

$$
\begin{gathered}
P_{3}(x)=P_{3}\left(x_{1}\right)=P_{3}\left(x_{2}\right)=P_{3}\left(x_{3}\right)=\ldots=P_{3}\left(x_{n}\right) \\
P_{3}(y)=\left(P_{3}\left(y_{1}\right)+P_{3}\left(y_{2}\right)+P_{3}\left(y_{3}\right)+\ldots+P_{3}\left(y_{n}\right)\right) / n
\end{gathered}
$$

$P_{4}$ can be acquired in the same way as $P_{3}$. For the back part of sole, similarly, there must be one point or several points lie in the most inner part of back sole. Thus, $P_{4}$ can also be acquired from Eq.7 and Eq.8.

$$
\begin{gathered}
P_{4}(x)=P_{4}\left(x_{1}\right)=P_{4}\left(x_{2}\right)=P_{4}\left(x_{3}\right)=\ldots=P_{4}\left(x_{n}\right) \\
P_{4}(y)=\left(P_{4}\left(y_{1}\right)+P_{4}\left(y_{2}\right)+P_{4}\left(y_{3}\right)+\ldots+P_{4}\left(y_{n}\right)\right) / n
\end{gathered}
$$

The last point $P_{5}$ of outline lies in the outer of sole. For the reason that the outer line of line is almost an oblique line in the static gait pressure matrix, the midpoint of this line can be regard as the characteristic point of outer sole. Different from the two inner points, the $\mathrm{x}$-coordinate and $\mathrm{y}$-coordinate of the midpoint are both the average value of outer line of sole. Thus the $P_{5}$ will be acquired as follow in Eq.9 and Eq.10:

$$
\begin{gathered}
P_{5}(x)=\left(P_{5}\left(x_{1}\right)+P_{5}\left(x_{2}\right)+P_{5}\left(x_{3}\right)+\ldots+P_{5}\left(x_{n}\right)\right) / n \\
P_{5}(y)=\left(P_{5}\left(y_{1}\right)+P_{5}\left(y_{2}\right)+P_{5}\left(y_{3}\right)+\ldots+P_{5}\left(y_{n}\right)\right) / n
\end{gathered}
$$

Although the outer line of sole cannot be a standard line, this line can be regard as a rough straight line, in other word, and the outer points of static gait pressure matrix can be treated as the outer line of sole.

After these orders, three points $P_{3}, P_{4}, P_{5}$ will be obtained and these three points can constitute three distances between each other. Therefore, $d_{3}, d_{4}, d_{5}$ will be calculated as follow, Eq. 11, 12, 13:

$$
\begin{gathered}
d_{3}=\sqrt{\left(P_{4}(y)-P_{5}(y)\right)^{2}+\left(P_{4}(x)-P_{5}(x)\right)^{2}} \\
d_{4}=\sqrt{\left(P_{3}(y)-P_{5}(y)\right)^{2}+\left(P_{3}(x)-P_{5}(x)\right)^{2}} \\
d_{5}=\sqrt{\left(P_{3}(y)-P_{4}(y)\right)^{2}+\left(P_{3}(x)-P_{4}(x)\right)^{2}}
\end{gathered}
$$

After $d_{1}, d_{2}, d_{3}, d_{4}, d_{5}$ are gained, the feature of static gait recognition is obtained. These five distances constitute the eigenvector $D=\left[d_{1} d_{2} d_{3} d_{4} d_{5}\right]$. Definitely, due to the difference between testers' footprint and pressure, each tester has their own static gait pressure matrixes. So different tester also have their own eigenvectors Ds which can be the recognition feature of tester's identity.

\subsection{Classifier selections}

The last step of recognition course is the recognizing by classifier. Through experiment, SVM proves to be the best classifier for the static gait recognition course ${ }^{[17]}$.

Since Vapnlik et al put forward the SVM (Support Vector Machine), this method had already solved the problem of nonlinearity and small sample and get wide using in classifier field. When processing the problem of nonlinearity filed, SVM uses a nonlinear transformation $\Phi$ to make samples map from input space into a certain high-dimensional space and then construct 
the optimal hyper plane. When the optimal hyper planes are creating, the training algorithm only involves the problem of inner product in the certain high-dimensional space so the key problem is to find the kernel function $\quad \boldsymbol{K}\left(x_{i}, x_{j}\right)=\left\langle\Phi\left(x_{i}\right), \Phi\left(x_{j}\right)\right\rangle \quad$. Besides, if $\boldsymbol{K}\left(x_{i}, x_{j}\right)$ meets the Mercy condition, this kernel function will correspond the inner product in the space transformation. In fact, the training problem is a convex quadratic optimization problem whose objective function describes as Eq.14 and Eq.15:

$$
\max H(a)=\sum_{i=1}^{N} a_{i}-\frac{1}{2} \sum_{i=1}^{N} \sum_{j=1}^{N} y_{i} y_{j} a_{i} a_{j} K\left(x_{i}, x_{j}\right)
$$

Subject to:

$$
\left\{\begin{array}{c}
0 \leq \alpha_{i} \leq L \quad i=0, \cdots, n \\
\sum_{i=1}^{n} y_{i} \alpha_{i}=0
\end{array}\right.
$$

$a_{i}, i=1,2 \cdots, N$ are lagrangian multiplier, $y_{i} \in\{1,-1\}$ are the category labels the $x_{i}$ correspond. $L$ is the upper bound of lagrangian multiplier. Then select the Gaussian Kernel Function: $k\left(x_{i}, x\right)=\exp \left(-\left\|x_{i}-x\right\|^{2} / \sigma^{2}\right)$ and using training samples to achieve optimal solution of $a_{i}$. After that, calculate the value of sign function $f(x)=\operatorname{sgn}\left(\sum_{i=1}^{N} y_{i} a_{i} K\left(x_{i}, x\right)+b\right)$ for each testing sample $\mathbf{x}$ in order to judge the classification of $\mathbf{x}$.

In most cases, SVM classifier is suitable for dichotomy classification. For the issue of multiclassification, it can be modify to suit the current course. This course can be divided into two kinds of methods: one-to- two classify and one- to- many classify. This paper chooses the former one. The one-to-two classify can also be called pairing classify, this classifier constructs two kinds of classifiers in $\mathrm{C}$ training samples. Here, every possible classifier takes its training course in the two kinds of training samples of $\mathrm{C}$ training samples. In this way, there will be $\mathrm{C}(\mathrm{C}-1) / 2$ classifiers come into beings. For instance, if the training sample can divide into classifiers between the No.i and No.j of classifier, the following question can be resolved.

$$
\begin{gathered}
\min _{\omega^{i j}, b^{i j}, \xi^{i j}} \frac{1}{2} \omega^{i j} \cdot \omega^{i j}+q \sum_{t=1}^{l} \xi^{i j} \\
\left\{\begin{array}{l}
\omega^{i j} \cdot \varphi\left(x_{t}\right)+b^{i j} \geq 1-\xi_{t}^{i j} \quad \text { If } y_{t}=i \\
\omega^{i j} \cdot \varphi\left(x_{t}\right)+b^{i j} \leq-1+\xi_{t}^{i j} \quad \text { If } y_{t} \neq i \\
\xi_{t}^{i j} \geq 0, i, j=1,2, \cdots, l .
\end{array}\right.
\end{gathered}
$$

For the $\mathrm{C}(\mathrm{C}-1) / 2$ classifiers, there must be $\mathrm{C}(\mathrm{C}-1) / 2$ decision functions and the decision function between the No.i and No. $\mathrm{j}$ is:

$$
f(x)=\operatorname{sgn}\left(\omega^{i j} \cdot \varphi\left(x_{t}\right)+b^{i j}\right)
$$

For the two kinds of classifiers, using suffrage method named using $C(C-1) / 2$ classifiers to classify the input samples $\mathbf{x}$ : If $\mathbf{x}$ belongs to the No.i category, then the No.i add one and also if $\mathrm{x}$ belongs to the No.j category, the No.j add one till all the categories are over. In the end, the category that gets more votes is the category the $\mathrm{x}$ belongs to.

\section{Experiment}

\subsection{Experiment data}

The data of this experiment come from actual measurement data of 50 testers whose bodies are suitable for the test, which means all testers have sound trunks. Their age range is from 23 to 26, the weights of males (29) are all around $65 \mathrm{~kg}$ and that of females (21) are all around $50 \mathrm{~kg}$ except for several special testers. During the course of data collecting, all testers are required to stand on pressure distribution beta with bare feet vertically. After that, a number of frames (about 8001200 different time duration has different number of frames. The longer time duration it takes the more frames we get) can be acquired. For each tester's frames, 10 successive frames will come to an average: the corresponding pressure points of the pictures of the 10 frames will be calculated and get an average value of each pressure point in the gait pressure matrix, thus 10 frames of gait pressure matrix data can be neutralized into 1 frame data. By means of this method, 130 frames of data (each 10 frames are continuous) will be made into 13 frames of data, which includes 10 gait pressure matrixes that are selected as the training set whose scale is $50 \times 10$, and the scale of other 10 frames selected as the testing set is $50 \times 3$. Then $50 \times 1$ will be choose as an experiment data every time therefore 3 experiment results : Test-1、Test-2、Test- 3 can be obtained.

\subsection{Experiment step}

(1) Obtain the training and testing sample using the static gait pressure matrix acquired by pressure distribution beta.

(2) With the processing of static gait pressure matrix, including eliminating all-zero lines and all-zero columns as well as the dispose of the issue of rotation invariance so as to insure all the static gait pressure matrixes get same processing course.

(3) Extract the feature of the static gait pressure matrix to process the recognition experiment.

(4) Compare the KNN classifier and SVM classifier to select the best classifier for the experiment. Finally, get the result of static gait recognition.

\subsection{Experiment result and analysis}

The feature of this paper is based on the combination of two distances of pressure points and three distances of outline points. Firstly, for the pressure points, if the static gait pressure matrix is divided into two parts, the result will be the best when compared with the pressure points of three or more parts of sole combined with three outline points. In addition, for the outline points, if three points of outline of static gait pressure matrix are selected, the result will be the best when compared with four of more points of outline of sole combined with two pressure points. The result of experiment can be seen in the Table 1. 
Table 1. Compared with similar methods

\begin{tabular}{|c|c|c|c|}
\hline Methods & Test-1 & Test-2 & Test-3 \\
\hline $\begin{array}{c}\text { Four outline } \\
\text { distance and } \\
\text { pressure distance }\end{array}$ & $46 / 50$ & $45 / 50$ & $44 / 50$ \\
\hline $\begin{array}{c}\text { Three outline } \\
\text { distance alone }\end{array}$ & $39 / 50$ & $41 / 50$ & $37 / 50$ \\
\hline $\begin{array}{c}\text { Three pressure distance } \\
\text { alone }\end{array}$ & $43 / 50$ & $45 / 50$ & $43 / 50$ \\
\hline Paper & $48 / 50$ & $47 / 50$ & $47 / 50$ \\
\hline
\end{tabular}

From table 1, apparently, three pressure distances combined with two outline distance is best of all and the reason will be showed as follow: If there are fewer points that is less distance from static gait pressure matrix, there will be less information for the feature. On the contrary, more points bring more mistake information of gait feature. So extracting the best number and position of points in static gait pressure matrix is a vital step of the whole course. Too many or too few points both have a bad influence on the recognition course. From the results of table 1, we can draw the conclusion that three outline distances and two pressure distanced constituted by three outline points and two pressure points are the best for feature extraction.

Since there are also some other traditional methods based on other features, it is necessary to compare the experiment of this paper with traditional methods. The results are showed in the table 2.

Table 2. Compared with different traditional methods

\begin{tabular}{|c|c|c|c|}
\hline Methods & Test-1 & Test-2 & Test-3 \\
\hline $\begin{array}{c}\text { Pressure point } \\
\text { coordinate }\end{array}$ & $33 / 50$ & $33 / 50$ & $37 / 50$ \\
\hline Square & $39 / 50$ & $32 / 50$ & $37 / 50$ \\
\hline $\begin{array}{c}\text { Distance between } \\
\text { partial pressure } \\
\text { point }\end{array}$ & $44 / 50$ & $46 / 50$ & $45 / 50$ \\
\hline Paper & $48 / 50$ & $47 / 50$ & $47 / 50$ \\
\hline
\end{tabular}

From table 2, we can see that the method in this paper performs better than other traditional methods. For the method based on overall-pressure point coordinate, the point of different testers may exists in the similar position because it can be influenced by many factors like weight, arch shape, sole shape and so forth so that they will make this point similar. As well as the method based on overall-pressure point coordinate, the method based on square has the same problem, namely, low recognition efficiency. Besides, for the method based on distances between partial points which divides whole sole into three part then tests the distances from the overall-point to three partial points, it also has a lower recognition efficiency than the method in this paper although it has already got a better result of recognition for the method in this paper has used more information of static gait pressure matrix.

The aforementioned result is based on SVM classifier. The next step is to test that compared with KNN classifier, whether SVM is the better one. The result can be seen in the table 3 .
Table 3. Results based on different classifier

\begin{tabular}{|c|c|c|c|}
\hline Methods & Test-1 & Test-2 & Test-3 \\
\hline KNN & $47 / 50$ & $44 / 50$ & $44 / 50$ \\
\hline SVM & $48 / 50$ & $47 / 50$ & $47 / 50$ \\
\hline
\end{tabular}

From table 3, slightly, the recognition based on SVM is little better than that on $\mathrm{KNN}$, so that in this paper, we select the SVM as the classifier.

\section{Conclusion}

This paper does the identity recognition course with the static gait recognition matrix got by the pressure distribution beta, through preprocessing course named the rotation invariance course and feature extraction, with the SVM classifier. The result of the experiment elects the better feature and classifier selection and shows the accuracy of recognition of testers. Moreover, the idea of static gait recognition can also be used in dynamic gait recognition. However, the shortage of the experiment lies in the selection of static gait pressure matrix that means half-baked or unstable static gait pressure footprints cannot be used in such method, which is exactly right the next step of this recognition course.

\section{Acknowledgements}

This work is supported by National Key R\&D Program of China (No. 2017YFC0803300)

\section{References}

1. J. A. Hanley and B.J. McNeil. The meaning and use of the area under a receiver operating characteristic (roc) curve. Radiology, 82(143):29-36, (1982)

2. P.S. Huang, C. J. Harris, and M. S. Nixon. Human gait recognition in canonical space using temporal templates. IEEE Procs. Vision Image and Signal Processing, 146(2):93-100, 1999.

3. J.J. Little and J.E. Boyd. Recognizing people by their gait: the shape of motion. Videre, 1(2), (1998)

4. S. Sarkar, P. Phillips, Z. Liu, I. Vega, P. Grother, and K. Bowyer, "The HumanID gait challenge problem: data sets, performance, andanalysis," IEEE Trans. Pattern Anal. Mach. Intell., vol. 27, no. 2, pp. 162-177, Feb. (2005)

5. M. Turk and A. Pentland, "Face recognition using eigenfaces," in Proc. IEEE Conf. Comput. Vision Pattern Recognit., pp. 586-591. (1991)

6. G.Veres, L. Gordon, J. Carter, and M. Nixon, "What image information is important in silhouette-based gait recognition?," in Proc. IEEE Conf.Comput. Vision Pattern Recognit., pp. 776-782. ,(2004)

7. Z. Liu and S. Sarkar, "Simplest representation yet for gait recognition:averaged silhouette," in Proc. IEEE Conf. Pattern Recognit., pp.211-214.(2004) 
8. GW. I Scholhorn, . Nigg B.M, D.J.Stephanshyn, andW. Liu, "Identification of individual walking patterns using time discrete and time continuous data sets," Gait and Posture, vol. 15, pp.180-186, (2002)

9. M.S. Nixon, J.N. Carter, J.M.Nash, P.S. Huang, D. Cunado, and S.V. Stevenage. Automatic gait recognition. In Motion Analysis and Tracking (Ref. No. 1999/103), IEE Colloquium on, pages 3/1-3/6, (1999)

10. S. Mowdray and M. Nixon, "Extraction and recognition of periodically deforming objects by continuous, spatio-temporal shape description," in Proc. IEEE Conf. Comput. Vision Pattern Recognit.,, pp.895-901(2004)

11. Ross Cutler and Larry Davis. Robust real-time periodic motion detection, analysis, and applications. IEEE Transactions on Pattern Analysis and Machine Intelligence, 22(8):781 -796, (2000)

12. C. Stauffer andW.E.L. Grimson. Adaptive background mixture models for real-time tracking. In CVPR, 1999.

13. R. Tanawongsuwan and A. Bobick, "Gait recognition from time-normalized joint-angle trajectories in the walking plane," in Proc. IEEE Conf. Comput. Vision Pattern Recognit., pp. 726731. (2001)

14. J. Han and B. Bhanu, "Statistical feature fusion for gait-based human recognition," in Proc. IEEE Conf. Comput. Vision Pattern Recognit, pp. 842-847. (2004)

15. D. Cunado, J.M. Nash, M.S. Nixon, and J. N. Carter, "Gait extraction and description by evidencegathering," Proc. of the International Conference on Audio and Video Based Biometric Person Authentication, pp.43-48,( 1995)

16. L. Lee and W.E.L. Grimson, "Gait analysis for recognition and classification," Proceedings of the IEEE Conference on Face and Gesture Recognition, pp. 155-161, (2002)

17. A. Kale, N. Cuntoor, and R. Chellappa, "A framework for activity-specific human recognition," Proceedings of the International Conference on Acoustics, Speech and Signal Processing (Orlando, FL), May (2002)

18. Yu-Feng Li. James T.Kwok. Zhi-Hua Zhou. CostSensitive Semi-Supervised Support Vector Machine. 